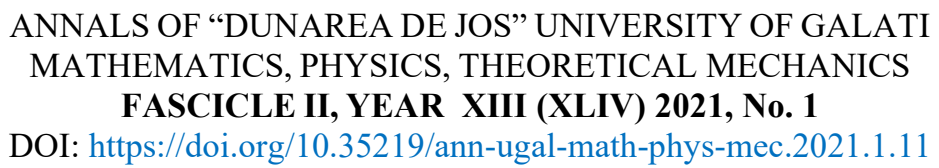

\title{
3D volume reconstruction of brain tissues using nonlinear filters, $k$-means clustering, and Bland-Altman analysis
}

\author{
Lenuța Pană ${ }^{1}$, Simona Moldoveanu ${ }^{2, *}$, Luminița Moraru, ${ }^{1}$, \\ 1 "Dunarea de Jos" University of Galati, Faculty of Sciences and Environment, 800008 Galati, Romania \\ 2 "Dunărea de Jos" University of Galați, Faculty of Automation, Computers Sciences, Electronics and Electrical \\ Engineering, 2 Stiinței Street, 800210, Galați, Romania \\ *Corresponding author: simona.moldovanu@ugal.ro
}

\begin{abstract}
This paper aims to provide a sound estimation of the true value and proportion of white matter (WM), gray matter (GM), and cerebrospinal fluid (CSF) of the brain DTI images for a proper 3D volume reconstruction. During the pre-processing stage, two nonlinear filters are operated, i.e. bilateral and anisotropic diffusion. The segmentation of each brain tissue is performed using the k-means clustering algorithm. To minimize filters bias and for obtaining the best reproducible results, a statistical analysis has been performed. Thus, the skewness and kurtosis statistics features were computed for each segmented brain tissue and filter. The fuzzy k-means method allows for clustering analysis and the Bland-Altman analysis investigates the agreement between two filtering techniques of the same statistics feature and brain tissue. Then the 3D reconstruction method is presented using ImageJ and the image stacks for raw and processed data. We conclude that anisotropic diffusion filter offers the best results and $3 \mathrm{D}$ reconstruction of brain tissues is feasible.
\end{abstract}

Keywords: brain tissues, K-clustering method, non-linear filters, statistics features, 3D reconstruction.

\section{INTRODUCTION}

In modern neuroscience, imaging techniques are used to study the structure of the brain. 2D segmentation of brain tissue in DTI (Diffusion Tensor Imaging) images represents an important tool for analyzing brain development [1], for the 3D visualization of a specific tissue or brain's anatomical structures [2], for delineating pathological regions, or for investigating brain changes. An ideal segmentation technique should meet some requirements such as minimal user interaction, fast calculation, and accurate and robust results [3].

There are various brain MRI segmentation methods having different accuracy and degree of complexity. Thresholding is a simple segmentation method and belongs to the intensity-based methods. It uses the intensity histogram to determine the best intensity values which separate the desired brain tissue classes. This technique does not take into account the spatial characteristics of an image and can provide misclassification due to random noise and complexity of the intensity distribution of brain tissues [4]. The most reliable segmentation method is fuzzy c-means (FCM) which is an iterative segmentation method. Shen et al. [5], proposed an extension of the fuzzy c-means clustering algorithm method by using both simulated and real MR brain images corrupted by various noise levels. Their method uses the relative location and features of neighboring pixels to improve the performance of the segmentation. Somasundaram and Kalaiselvi [6] used k-means, Fuzzy C Means, and Expectation Maximization segmentation methods to correctly identify the white matter, gray matter, cerebrospinal fluid in T1w and T2w MRI images. K-means algorithm was shown to better classify the white matter, the Fuzzy C Means method misclassified gray matter and cerebrospinal fluid while the Expectation Maximization algorithm correctly classifies white matter, gray matter, 
cerebrospinal fluid. A hybrid method based on gray level co-occurrence matrix (GLCM) and supportvector machine (SVM) for brain MRI segmentation has been proposed by Yazdani et al. [4]. The performance of this hybrid method indicates a Kappa similarity index of 91.5 which is an improvement of the overall segmentation performance.

Usually, 2D and 3D images are used to visualize anatomical and pathological components of the brain (tumor, brain surface, skull, arteries, and veins). Nowadays, surgeons ask for a clear and precise 3D brain image during complex robotic surgery [2]. Kikinis et al. [7], used 2D MRI brain images with tumors and vascular malformation for a 3D reconstruction by rendering surface Various spatial information about cortex, gray matter, white matter, blood vessels, tumors can be obtained with greater fidelity and further, the planning process of the surgery can be improved. Using neurosurgical planning software Mert et al. [8] used a 3D brain surface visualization technique realized to reconstruct the $3 \mathrm{D}$ brain surface from $2 \mathrm{D}$ brain images $\mathrm{RMN}$. The topographic lesion localization was precise and fast compared to the 2D images. Harput et al. [9] performed a 3D reconstruction of the cerebral surface from 2D brain RMN from patients with neocortical lesions, using the free software called OsiriX. The method allowed an accurate identification of the tumor's borders.

In this paper, bilateral and anisotropic diffusion filters are used during the pre-processing stage. The k-means clustering algorithm performs the segmentation of the brain tissues into the DTI filtered images. The histogram characterization allows the extraction of more informative features like skewness and kurtosis. They are used to better capture the properties of tissues, to minimize filters bias, and for best reproducible results. The fuzzy k-means and the Bland-Altman methods provide a sound clustering analysis and assess the agreement between two filtering techniques of the same statistics feature and brain tissue. The 2D datasets (both raw and filtered images) are used for a 3D reconstruction, with the instrumentality of the free software ImageJ, with the final goal to obtain images that closely approximate the original studied structure.

The remaining sections of the paper are as follows: the mathematical methods, the datasets, image acquisition, and post-processing are provided in Section 2; Section 3 provides the results and discusses, and Section 4 concludes the paper.

\section{METHODS \\ 2.1. MATHEMATICAL APPROACHES}

The anisotropic diffusion filter (ADF)

$\mathrm{ADF}$ is used to adaptively remove the noise, maintaining the image edges $[10,11]$ :

$$
\begin{aligned}
& \frac{\partial I}{\partial t}=\nabla(D \nabla I) \\
& D=\left(\begin{array}{ll}
a & b \\
b & c
\end{array}\right)
\end{aligned}
$$

where I denote the brain DTI image, $\mathrm{t}$ is the diffusion time, $\nabla$ is the gradient operator, $\mathrm{D}$ is the diffusion tensor and it is adapted to the local brain structure. The diffusion tensor D uses the same eigenvectors as the structure tensor. The eigenvalues of $\mathrm{D}$ are [10]: .

$$
\begin{aligned}
& \lambda_{1}:=c_{1} \\
& \lambda_{2}:=\left\{\begin{array}{c}
c_{1} \text { if } \mu_{1}=\mu_{2} \\
c_{1}+\left(1-c_{1}\right) \exp \left(\frac{-c_{2}}{\left(\mu_{1}-\mu_{2}\right)^{2}}\right)
\end{array}\right.
\end{aligned}
$$

where $0<\mathrm{c}_{1}<<1$ and $\mathrm{c}_{2}>0$. 
The elements of $D$ are as follows:

$$
\begin{aligned}
& a=\lambda_{1} \cos ^{2} \alpha+\lambda_{2} \sin ^{2} \alpha \\
& b=\left(\lambda_{1}-\lambda_{2}\right) \sin \alpha \cos \alpha \\
& c=\lambda_{1} \sin ^{2} \alpha+\lambda_{2} \cos ^{2} \alpha
\end{aligned}
$$

By using $\gamma_{1}$ and $\gamma_{2}$, the eigenvectors of the structure tensor, the smoothing directions are determined. The eigenvalues $\mu_{1}$ and $\mu_{2}$ define the contrast along with the considered directions. When $\mu_{1}>>\mu_{2}$, the eigenvector $\gamma_{1}$ indicates the orientation with the highest intensity fluctuation and $\gamma_{2}$ is the direction for smoothing.

\section{Bilateral filtering}

A bilateral filter reduces the noise, preserves the edges by weighting the sum of the pixels in a local neighborhood. The used weights are computed based on the spatial distance and the intensity distance. The bilateral filter BF[ ] for an image $I$ is defined_[12] :

$$
\mathrm{BF}[I]_{p}=\frac{1}{W_{p}} \sum_{q \in S} G_{\sigma_{S}}(\|p-q\|) G_{\sigma_{R}}\left(\left|I_{p}-I_{q}\right|\right) I_{q}
$$

where $W_{\mathrm{p}}$ is a normalization factor:

$$
W_{p}=\sum_{q \in S} G_{\sigma_{S}}(\|p-q\|) G_{\sigma_{R}}\left(\left|I_{p}-I_{q}\right|\right)
$$

$\sigma_{\mathrm{s}}$ is a spatial parameter; $\sigma_{\mathrm{R}}$ a range parameter; $I_{p}$ and $I_{q}$ denote the intensity values of the pixels $p$ and $q$, respectively; $G_{\sigma_{s}}$ is a spatial Gaussian weight; $G_{\sigma_{r}}$ is a range Gaussian who modulates the $I_{q}$ influence when its intensity is different from $I_{p} ; S$ is the spatial domain and $\mathrm{R}$ is the range domain. $\|p-q\|$ is the Euclidean distance between pixel $p$ and pixel $q$.

\section{K-means algorithm}

$\mathrm{K}$-means is a clustering algorithm able partitioning data into $k=n$ clusters of similar objects. For our study, $k=3$ (i.e., $k=1$ is for CSF, $k=2$ for $\mathrm{WM}$, and $k=3$ for $\mathrm{GM}$ ).

The k-means is built on an expectation-maximization algorithm and it tries to minimize distances within a cluster and maximize the distance between different clusters [13, 14]:

$$
\mathbf{J}=\sum_{j=1}^{k} \sum_{i=1}^{n}\left\|x_{i}^{(j)}-c_{j}\right\|^{2}
$$

where $\left\|x_{i}^{(j)}-c_{j}\right\|^{2}$ is the Eucledian distance between a data point, $x_{\mathrm{i}}^{(\mathrm{j})}$, and a cluster center, $c_{j}$, iterated over $\mathrm{i}^{\text {th }}$ all $\mathrm{k}$ points in the cluster; $\mathrm{n}$ is data points.

\section{Fuzzy K-means algorithm}

This algorithm differs from K-means through use of weighted squared errors [15]. The fuzzy k-means based on [16] 


$$
\mathbf{J}=\sum_{j=1}^{k} \sum_{i=1}^{n} u_{i j}^{m}\left\|x_{i}^{(j)}-c_{j}\right\|^{2}
$$

where $u_{i j}$ is the membership value of $j^{\text {th }}$ data point to $i^{\text {th }}$ cluster, and meets the following conditions:

$$
0 \leq u_{i j} \leq 1 \quad \sum_{i=1}^{n} u_{i j}=1 \quad 0<\sum_{j=1}^{k} x_{j}<n
$$

$m>1$ is a weighting exponent or fuzzifier parameter.

Kurtosis and Skewness

The kurtosis measures the peakedness (or the heaviness of the tails) of the distribution [17, 18]:

$$
\gamma_{1}=E\left[\left(\frac{X-\mu}{\sigma}\right)^{3}\right]=\frac{\frac{1}{L^{2}} \sum_{x, y}(I(x, y)-\mu)^{3}}{\sigma^{3}}
$$

The skewness measures the asymmetry of the distribution $[15,16]$ :

$$
\gamma_{2}=E\left[\left(\frac{X-\mu}{\sigma}\right)^{4}\right]-3=\frac{\frac{1}{L^{2}} \sum_{x, y}(I(x, y)-\mu)^{4}}{\sigma^{4}}-3
$$

$I$ is an image with dimension $L \times L, \mu$ is mean the $1^{\text {st }}$ moment, $\sigma$ is standard deviation, square of the $2^{\text {nd }}$ central moment and are defined as [17]:

$$
\begin{aligned}
& \mu=E[X]=\frac{1}{L^{2}} \sum_{x, y} I(x, y) \\
& \sigma=\sqrt{E\left[(X-\mu)^{2}\right]}=\sqrt{\frac{1}{L} \sum_{x, y}(I(x, y)-\mu)^{2}}
\end{aligned}
$$

\section{Brain volume computation}

2D image stacks generated for each segmented brain tissue are used for $3 \mathrm{D}$ reconstruction. To perform this task, the software ImageJ Plugin-3D-Volume Viewer is used. The volume is computed as [18]:

$$
V=h \times \sum_{k=1}^{N-1} A_{k}+A_{1} \times \frac{\delta}{2}+A_{N} \times \frac{\delta}{2}
$$

where $N=100$ is the number of the images for each segmented brain tissue in the stack; $h=1.5$ pixels is the distances between two successive slices in the stack; $A_{i}$ and $A_{N}$ are the area of the first and last image in the stack, respectively; $A_{k}$ denotes the area of the $k^{\text {th }}$ slice and $\delta$ the thickness of a slice.

\section{The Bland Altman method}

Generally, the Bland-Altman method assesses the agreement between two quantitative measurements. In the proposed study, the agreement between the bilateral and anisotropic diffusion filters is evaluated. This method asks for a limit of agreement of $95 \%$ of the data points placed within $\pm 2 \mathrm{SD}$ of the mean difference. The standard 
deviation SD indicates how accurate is the mean $(\mathrm{M})$ of the kurtosis and skewness, for each brain tissues $\mathrm{x}_{\mathrm{i}}$ [1921],

$$
S D=\sqrt{\frac{\sum_{i=1}^{N}\left(x_{i}-M\right)^{2}}{(N-1)}}
$$

\section{The Pearson coefficient}

The Pearson correlation coefficient indicates the relationships between the two methods [19-22]:

$$
r_{x y}=\frac{\sum_{i=1}^{N}\left(d_{i}-\bar{d}\right)\left(e_{i}-\bar{e}\right)}{\sqrt{\sum_{i=1}^{N}\left(d_{i}-\bar{d}\right)^{2}} \sqrt{\sum_{i=1}^{N}\left(e_{i}-\bar{e}\right)^{2}}} \quad \bar{d}=\frac{1}{N} \sum_{i=1}^{N} d_{i} \quad \bar{e}=\frac{1}{N} \sum_{i=1}^{N} e_{i}
$$

\subsection{SUBJECTS, IMAGE ACQUISITION AND POST-PROCESSING}

The programming environments are MATLAB R2017a, Image Processing toolbox, ImageJ plugin and MedCalc, respectively. This study includes 100 DICOM brain images of a healthy patient $(128 \times 128$ pixels) that were acquired without diffusion gradients $(\mathrm{b} 0=0 \mathrm{~s} / \mathrm{mm} 2)$ using a $1.5 \mathrm{~T}$ MRI scanner Philips Medical Systems, Best, Netherlands. The flow chart is shown in Fig. 1.

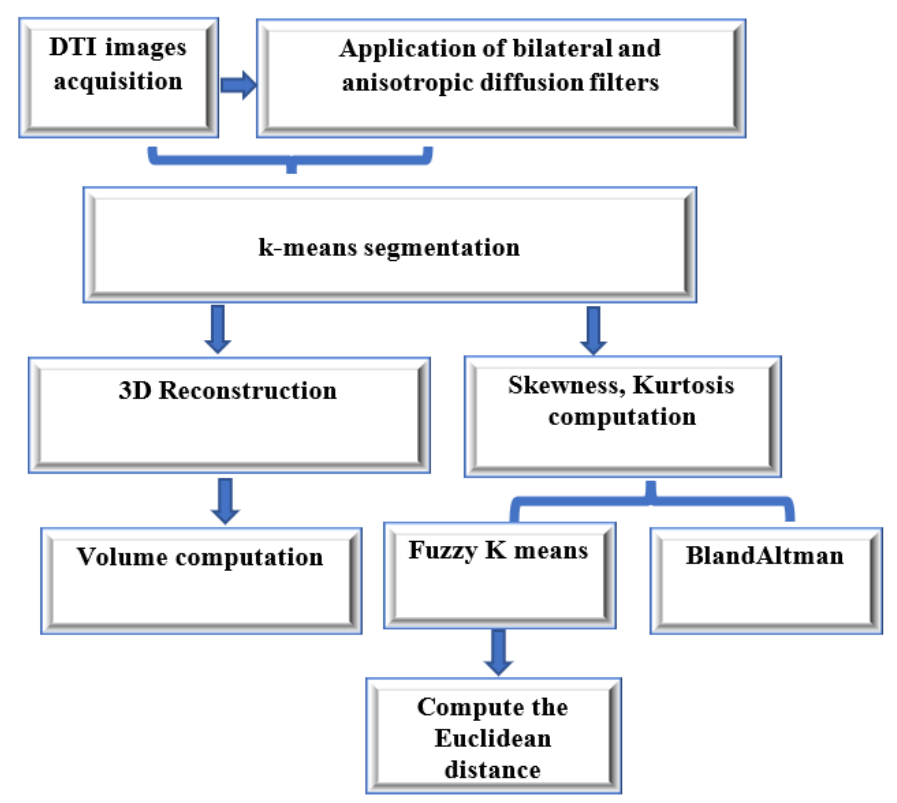

Fig. 1. The flow chart of the proposed method.

\section{RESULTS AND DISCUSSION}

The results of the k-means segmentation and separation process are shown in Fig. 2. 


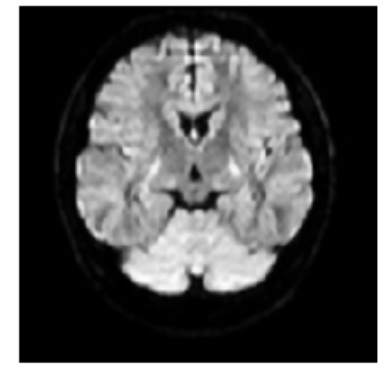

a)

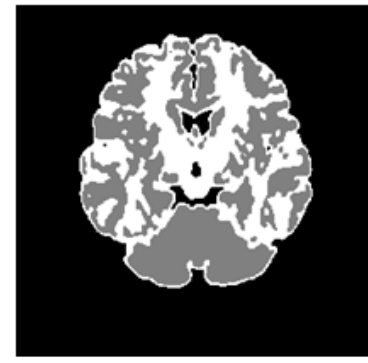

b)

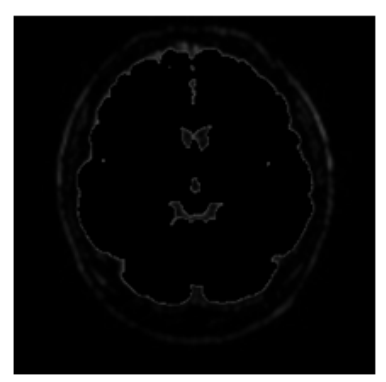

c)

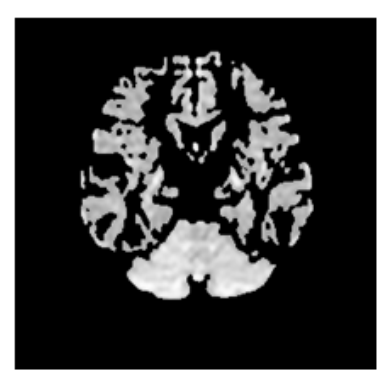

d)

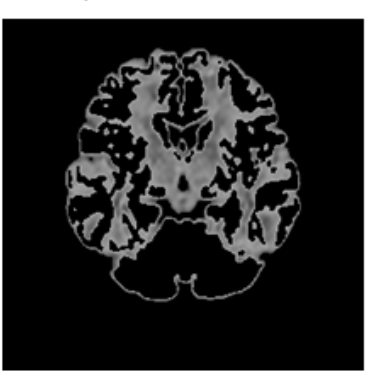

e)

Fig. 2. example of k-means segmentation and brain tissues separation process. a) anisotropic diffusion filter; b) clustering results; c) cluster 1 is for CSF; d) cluster 2 is for WM; e) cluster 3 is for GM.

The statistical descriptors kurtosis and skewness of the tissues contained in the MRI brain images are analyzed using the fuzzy k-means algorithm. The clustering operation has been performed for both filters. Figures 3 and 4 show the clustering results for kurtosis and skewness data, respectively.

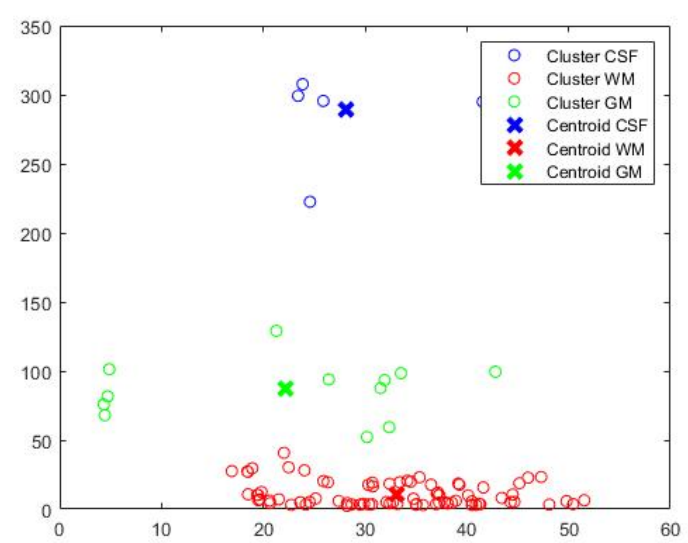

a)

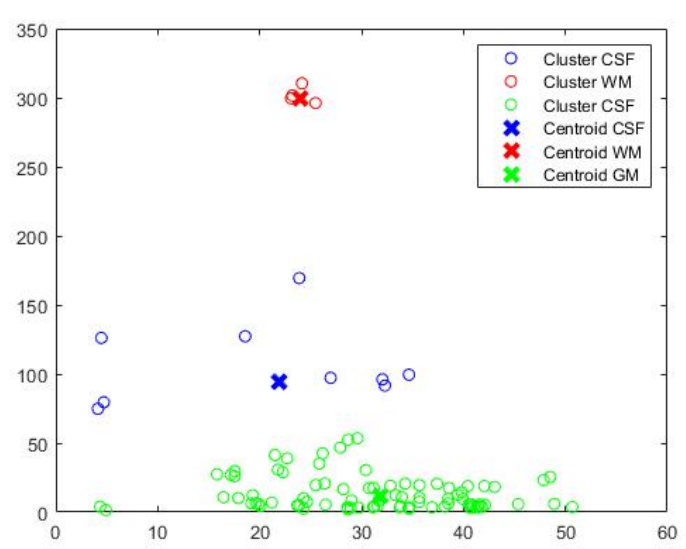

b)

Fig. 3. Clustering results for kurtosis values and for each brain tissue. a) the bilateral filter, b) anisotropic diffusion filter 


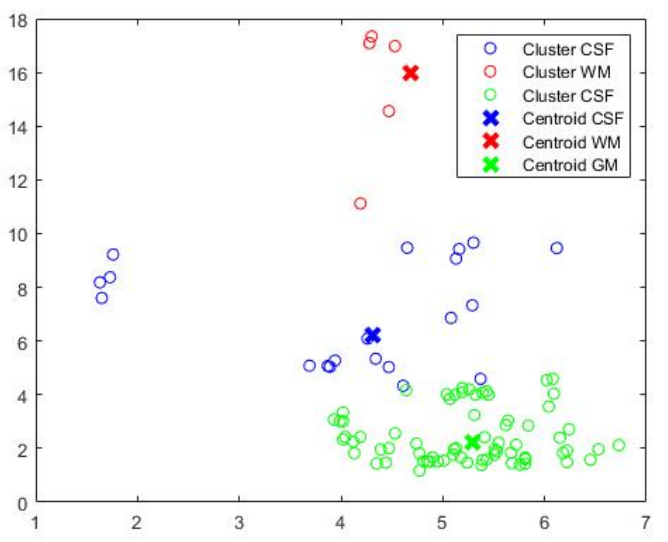

a)

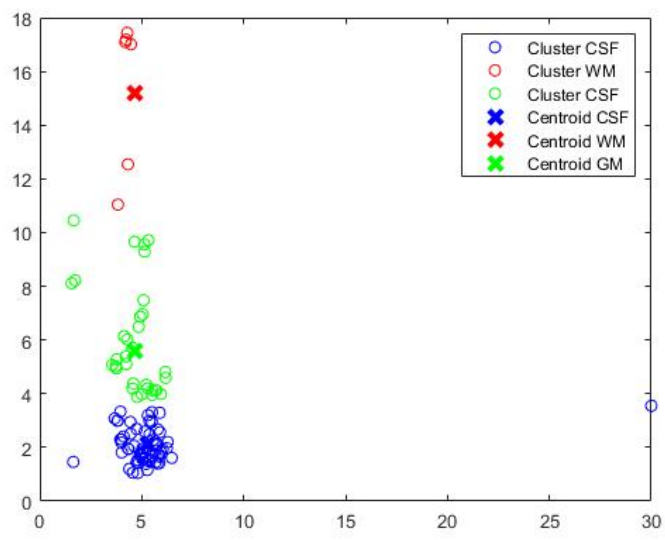

b)

Fig. 4. Clustering results for skewness values and for each brain tissue. a) the bilateral filter, $b$ ) anisotropic diffusion filter

The Euclidean distances between the center of the determined clusters are presented in Table 1. A greater distance between clusters indicates a greater dissimilarity between analyzed data.

Table 1 Distance between final cluster centers for kurtosis and skewness features

\begin{tabular}{|c|c|c|c|c|c|}
\hline Types of nonlinear filters & Features & & Euclidean & stance & \\
\hline \multirow{8}{*}{ Bilateral filter } & \multirow[t]{4}{*}{ Kurtosis } & & CSF & WM & GM \\
\hline & & CSF & - & 269.1137 & 68.53895 \\
\hline & & WM & 269.1137 & - & 262.2255 \\
\hline & & GM & 68.53895 & 262.2255 & - \\
\hline & \multirow[t]{4}{*}{ Skewness } & & CSF & WM & GM \\
\hline & & $\mathrm{CSF}$ & - & 10.58244 & 3.244547 \\
\hline & & WM & 10.58244 & - & 10.99234 \\
\hline & & GM & 3.244547 & 10.99234 & - \\
\hline \multirow{8}{*}{$\begin{array}{l}\text { Anisotropic diffusion } \\
\text { filter }\end{array}$} & \multirow[t]{4}{*}{ Kurtosis } & & CSF & WM & GM \\
\hline & & CSF & - & 75.17172 & 276.6298 \\
\hline & & WM & 75.17172 & - & 285.2366 \\
\hline & & GM & 276.6298 & 285.2366 & - \\
\hline & \multirow[t]{4}{*}{ Skewness } & & CSF & WM & GM \\
\hline & & CSF & - & 9.048268 & 2.936416 \\
\hline & & WM & 9.048268 & - & 9.275849 \\
\hline & & GM & 2.936416 & 9.275849 & - \\
\hline
\end{tabular}

Based on the clustering analysis represented in Figures 3 and 4 and data in Table 1, the following observations can be systematized:

- $\quad$ kurtosis better separates WM from both GM and CSF, as the Euclidean distances between the WM and CSF centroids is 269.1137 and between GM and WM is 262,2255 , respectively, for bilateral filter;

- $\quad$ kurtosis better separates GM from WM and CSF, as the Euclidean distances between the centroids of the GM and CSF is 276.6298 and between GM and WM is 285,2366, respectively, for anisotropic diffusion filter;

- $\quad$ skewness feature indicates a low degree of membership during the clustering operation for all brain tissues and for both filters.

However, there is no clear difference between the performance of the utilized filters. A further analysis based on Bland Altman's statistical interpretation of the data for each brain tissue is 
performed using MedCalc software. The agreement between two quantitative measurements provided by bilateral and anisotropic diffusion filters is displayed in Figures 5 and 6.

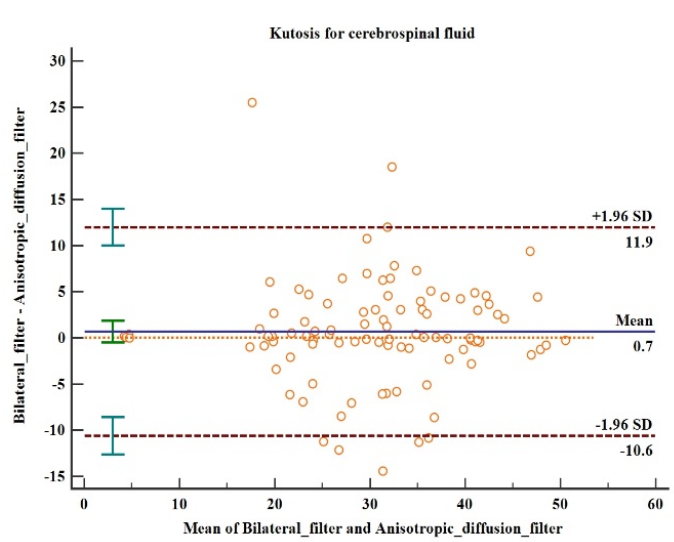

a) CSF results measured by the bilateral filter method may be 10.6 units below or 11.9 above method anisotropic diffusion filter. The width of the interval is 22.5 .

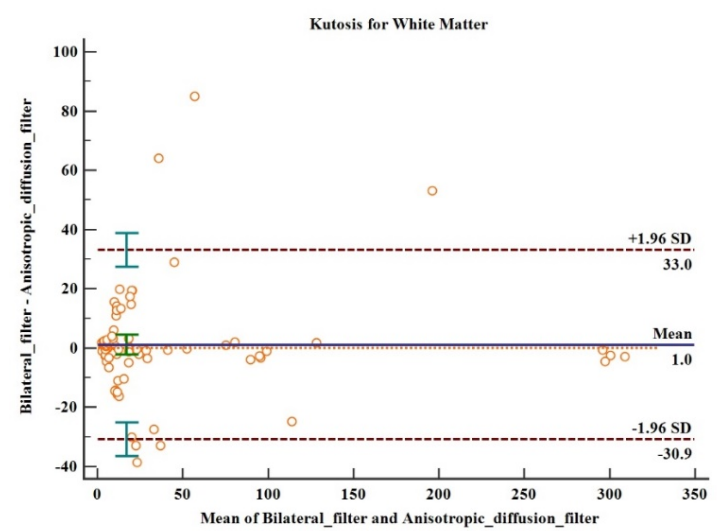

b) WM results measured by the bilateral filter method may be 30.9 units below or 33.0 above method anisotropic diffusion filter. The width of the interval is 63.9.

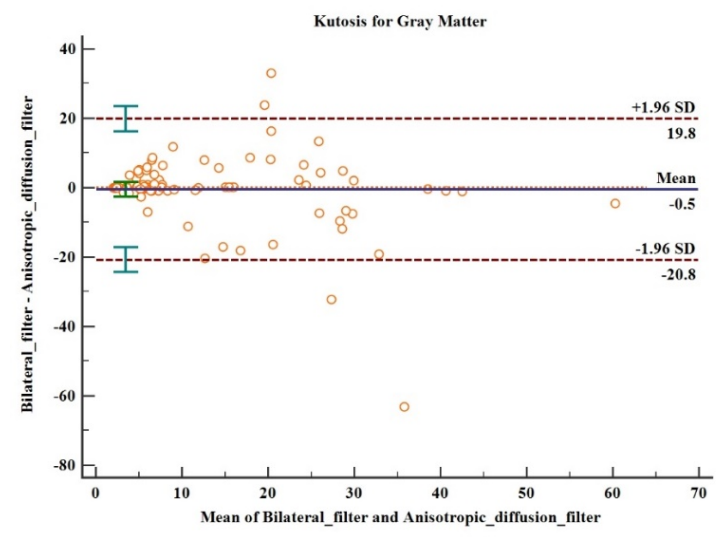

c) GM results measured by the bilateral filter method may be 20.8 units below or 19.8 above method anisotropic diffusion filter. The width of the interval is 40.6 .

Fig. 5. The Bland Altman plots for bilateral and anisotropic diffusion filters for kurtosis feature. Ox shows the average of two measurements provided by the bilateral and anisotropic diffusion filters. Oy indicates the differences between the methods' estimates. The confidence intervals for the mean difference and the agreement limits are indicated. The estimated bias is indicated by the mean difference and the fluctuations around this mean are provided by $S D$ 


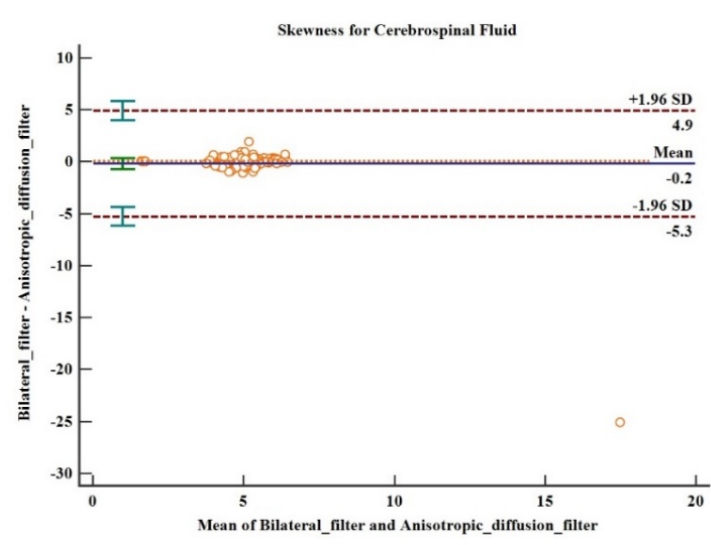

a) CSF results measured by the bilateral filter method may be 5.3 units below or 4.9 above method anisotropic diffusion filter. The width of the interval is 10.5 .

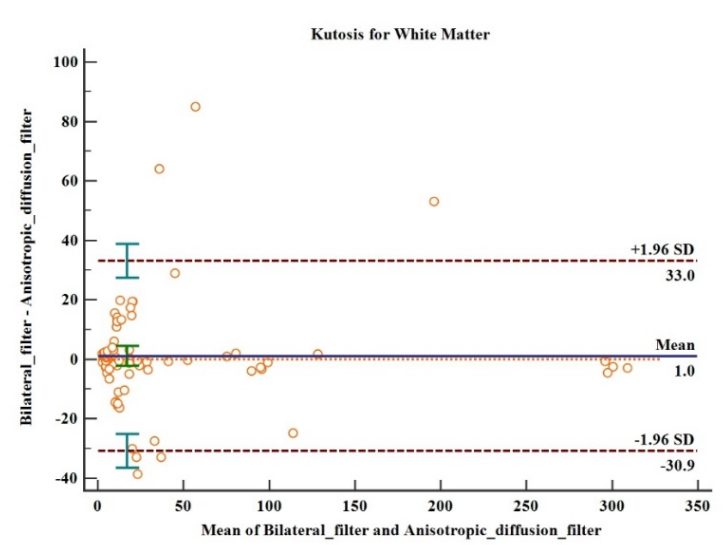

b) WM results measured by the bilateral filter method may be 2.8 units below or 3.1 above method anisotropic diffusion filter. The width of the interval is 5.9.

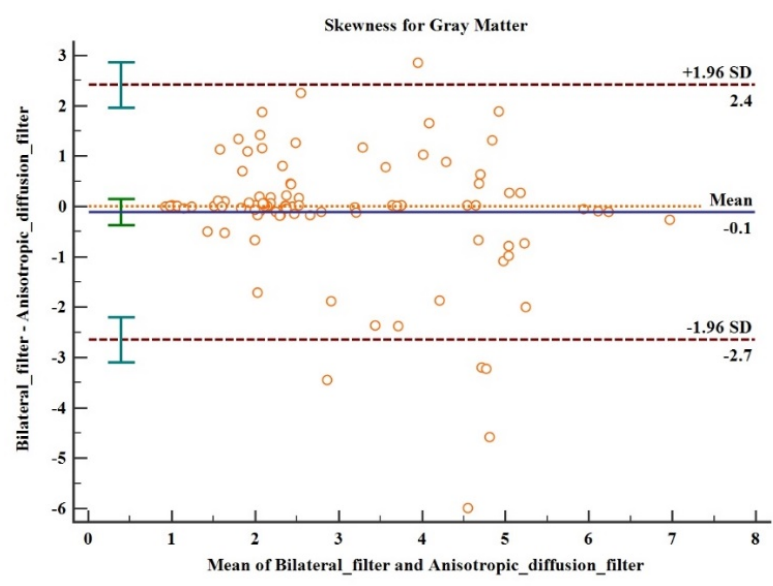

c) GM results measured by the bilateral filter method may be 2.7 units below or 2.4 above method anisotropic diffusion filter. The width of the interval is 5.1 .

Fig.6. The Bland Altman plots for bilateral and anisotropic diffusion filters for skewness features. Ox shows the average of two measurements provided by the bilateral and anisotropic diffusion filters. $O y$ indicates the differences between the methods' estimates. The confidence intervals for the mean difference and the agreement limits are indicated. The estimated bias is indicated by the mean difference and the fluctuations around this mean are provided by SD.

Information extracted from kurtosis data for CSF (Fig. 5a) and GM (Fig. 5c) indicates a good agreement between measurements of the two filters as the average difference is closer to zero.

For skewness data, the plot for WM (Fig. 6b) indicates also a good agreement between measurements of the two filters. Moreover, a concentrated distribution of CSF data (Fig. 6a) is observed on both sides of the median / bias. In this case, the limits of the agreement intervals are narrow, which indicates that both preprocessing methods are comparable.

To evaluate the relationship between the filtering methods, the Pearson correlation coefficients are computed. The results are presented in Tables 2 and 3 for kurtosis and skewness features, respectively. 
Table 2. The Bland Altman statistics ( $\mathrm{M} \pm 1.96 * \mathrm{SD}$, Bias and Pearson correlation) for kurtosis feature. $\mathrm{M}$ denotes the average of the differences between the measurements and SD is the standard deviation of the measured values

\begin{tabular}{|c|c|c|c|c|}
\hline Brain tissue & $\mathrm{M}+1.96^{*} \mathrm{SD}$ & $\mathrm{M}-1.96^{*} \mathrm{SD}$ & Bias & $\begin{array}{c}\text { Pearson } \\
\text { correlation } \\
\text { coefficient (r) }\end{array}$ \\
\hline CSF & 11.9 & -10.6 & 0.7 & 0.84 \\
\hline WM & 33 & -30.9 & 1 & $\mathbf{0 . 9 6}$ \\
\hline GM & 19.8 & -20.8 & -0.5 & 0,65 \\
\hline
\end{tabular}

Table 2 shows that a correlation is higher than 0.75 for CSF and WM so that the results provided by both filters show a good correlation. In the case of WM, a correlation coefficient close to the value 1 was determined indicating a linear relationship between both filters' output. There is also a negative average bias (i.e., a systematic negative error) related to the GM measurements.

Table 3. The Bland Altman statistics ( $\mathrm{M} \pm 1.96 * \mathrm{SD}$, Bias and Pearson correlation) for skewness feature. M denotes the average of the differences between the measurements and SD is the standard deviation of the measured values

\begin{tabular}{|c|c|c|c|c|}
\hline Brain tissue & M+1.96*SD & M-1.96*SD & Bias & $\begin{array}{c}\text { Pearson } \\
\text { correlation } \\
\text { coefficient }(r)\end{array}$ \\
\hline CSF & 4.9 & -5.3 & -0.2 & 0.31 \\
\hline WM & 2.4 & -2.7 & -0.1 & $\mathbf{0 . 9 1}$ \\
\hline GM & 3.1 & -2.8 & $\mathbf{0 . 1}$ & 0.66 \\
\hline
\end{tabular}

Table 3 shows a correlation coefficient close to the value 1 indicating a linear relationship between both filters' output related to WM and skewness feature. A good correlation was determined for GM, while for CSF the correlation is weak. On the other hand, for the skewness feature, the average bias (i.e. a systematic negative error) shows small values for all brain tissues.

Once the brain tissues are accurately extracted, we perform a 3D reconstruction to reveal brain tissue patterns with the preservation of local spatial relationships. The performance of both bilateral and anisotropic diffusion filters is assessed for brain volume quantification. The accurate 3D brain reconstructions can be used for quantitative brain morphometric analyses. Figures 6, 7, and 8 show the $3 \mathrm{D}$ reconstruction results and the quantifications of volumes data are displayed in Table 4. 


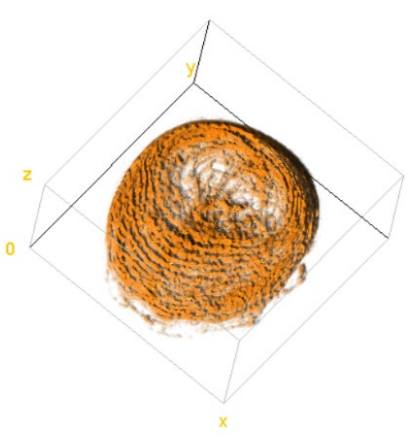

a)

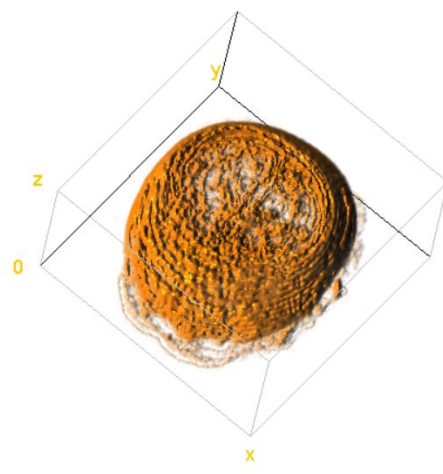

b)

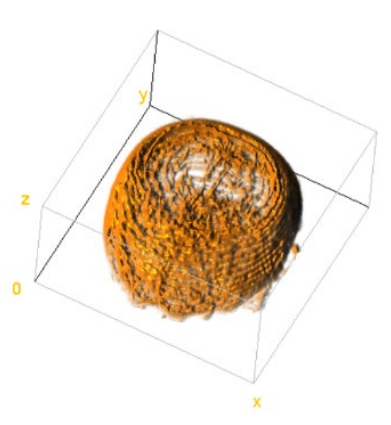

c)

Fig. 7. 3D reconstruction of the CSF brain tissue. (a) imaged volume from the raw dataset; (b) imaged volume from the bilateral filter dataset; (c) imaged volume from the anisotropic filter dataset.

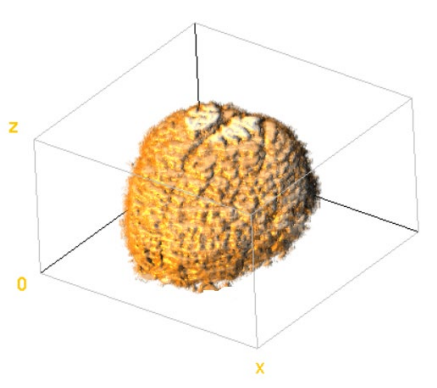

a)

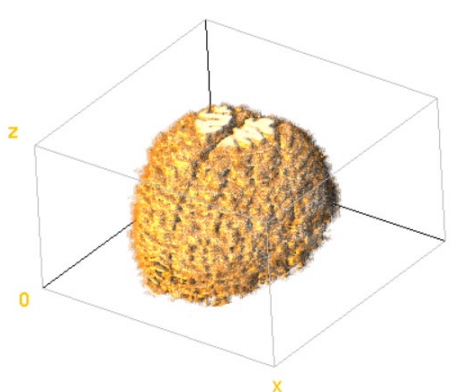

b)

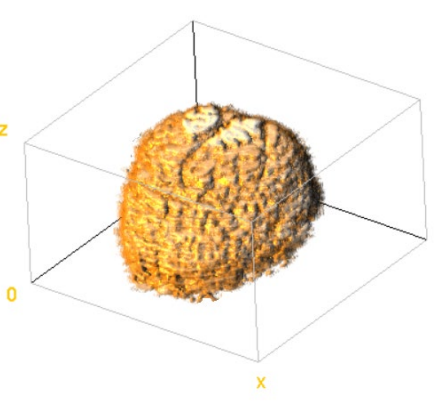

c)

Fig. 8. 3D reconstruction of the WM brain tissue. (a) imaged volume from the raw dataset; (b) imaged volume from the bilateral filter dataset; (c) imaged volume from the anisotropic filter dataset.

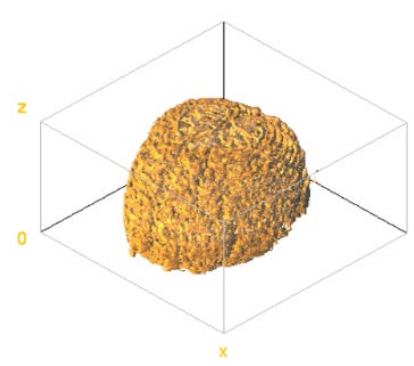

a)

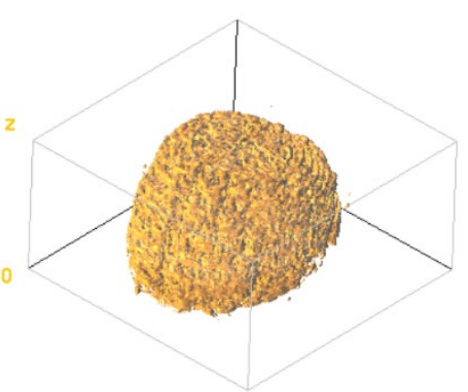

b)

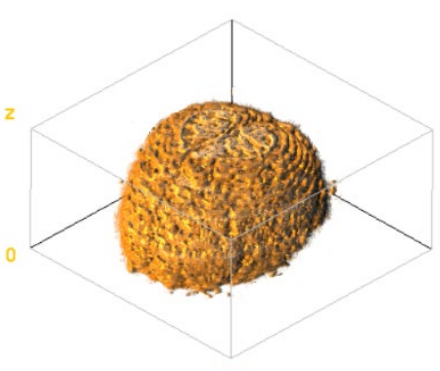

c)

Fig. 9. $3 D$ reconstruction of the GM brain tissue. (a) imaged volume from the raw dataset; (b) imaged volume from the bilateral filter dataset; (c) imaged volume from the anisotropic filter dataset.

Table 4. Volume values for each brain tissue

\begin{tabular}{|l|c|c|c|}
\hline & $\begin{array}{c}\text { CSF } \\
\left(\times 10^{6} \text { voxels }\right)\end{array}$ & $\begin{array}{c}\text { WM } \\
\left(\times 10^{6} \text { voxels }\right)\end{array}$ & $\begin{array}{c}\text { GM } \\
\left(\times 10^{6} \text { voxels }\right)\end{array}$ \\
\hline raw data & 0.18 & 0.55 & 0.76 \\
\hline bilateral filter data & 0.23 & 0.76 & 0.79 \\
\hline $\begin{array}{l}\text { anisotropic diffusion filter } \\
\text { data }\end{array}$ & $\mathbf{0 . 2 1}$ & $\mathbf{0 . 5 8}$ & $\mathbf{0 . 7 4}$ \\
\hline
\end{tabular}


The brain volume variation is small and positive for the anisotropic diffusion filter and is larger for the bilateral filter.

As a result, when we pool data from our studies, we can conclude that the anisotropic diffusion filter is a better solution for $3 \mathrm{D}$ reconstruction and volumetric analysis.

\section{CONCLUSIONS}

In this paper, two filters, namely the anisotropic diffusion filter and bilateral filter, and two statistical features (i.e. kurtosis and skewness) were investigated to assess their capability for an accurate 3D brain reconstruction. The coupling between the kurtosis feature and anisotropic diffusion filter has been found as the best tool for pre-processing, segmentation, and 3D brain tissues reconstruction.

The proposed method for 3D brain reconstruction and volume estimation offers a good foundation for further studies of different brain regions 3D reconstruction.

\section{References}

1. Despotović I., Goossens B., Philips W., MRI Segmentation of The Human Brain: Challenges, Methods, And Applications, Computational and Mathematical Methods in Medicine, 2015 (2015), Article Id 450341, 23 pages, doi: 10.1155/2015/450341.

2. Neyaz Z., Phadke R.V., Singh V., Godbole C., Three-dimensional visualization of intracranial tumors with cortical surface and vasculature from routine MR sequences, Neurol India, 65(2) (2017) 333-340.

3. Moraru L., Moldovanu S., Dimitrievici L. T., Dey N., Ashour A. S., Shi F., Fong S. J., Khan S., Biswas A., Gaussian mixture model for texture characterization with application to brain DTI images, Journal of Advanced Research, 16 (2019) 15-23, doi: 10.1016/j.jare.2019.01.001.

4. Yazdani S., Yusof R., Karimian A., Hossein Riazi A., Brain Tissue Classification in Magnetic Resonance Images, Jurnal Teknologi (Sciences \& Engineering), 72(2) (2015) 29-32.

5. Shen S., Sandham W., MRI Fuzzy Segmentation of Brain Tissue using Neighborhood Attraction with Neural-Network Optimization, IEEE Transactions on Information Technology in Biomedicine, 9(3) (2005) 459-467.

6. Somasundaram K., Kalaiselvi T., A Comparative Study of Segmentation Techniques used for MR Brain Images, Conference: Proceedings of the 2009 International Conference on Image Processing, Computer Vision, \& Pattern Recognition, Las Vegas, Nevada, USA, 2 (2019).

7. Kikinis R., Gleason P.L., Moriarty T.M., Moore M., Alexander E., Stieg P.E., Matsumae M., Lorensen W.E., Cline H.E., Black P.M., Jolesz F.A., Computer-assisted interactive threedimensional planning for neurosurgical procedures, Neurosurgery, 38(4) (1996) 640-649.

8. Mert A., Buehler K., Sutherland G.R., Tomanek B., Widhalm G., Kasprian G., Knosp E., Wolfsberger S., Brain tumor surgery with 3-dimensional surface navigation, Neurosurgery, 71(2 Suppl Operative):ons286-94; discussion ons294-5. doi: 10.1227/NEU.0013e31826a8a75. PMID: 22843134 (2012).

9. Harput M.V., Gonzalez-Lopez P., Türe U., Three-dimensional reconstruction of the topographical cerebral surface anatomy for presurgical planning with free OsiriX Software, Operative Neurosurgery, 10(3) (2014) 426-435.

10. Scharr H. and Weickert J., An Anisotropic Diffusion Algorithm with Optimized Rotation Invariance, In: Sommer G., Krüger N., Perwass C. (eds) Mustererkennung 2000. Informatik aktuell. Springer, Berlin, Heidelberg, pp 460-467, 2000, https://doi.org/10.1007/978-3-64259802-9 58.

11. C. Smochină, Tehnici de procesare a imaginii și de evaluare a segmentării, Iași, 2011.

12. Paris S., Kornprobst P., Tumblin J., Durand F., Bilateral Filtering: Theory and Applications, Foundations and Trends R in Computer Graphics and Vision, 4(1) (2008) 1-73. 
13. Pana L., Moldovanu S., Moraru L., Dey N., Ashour A.S., Brain tissue evaluation based on skeleton shape and similarity analysis between hemispheres, MDPI, Journals Computation, 8(2) (2020) https://doi.org/10.3390/computation8020031 .

14. Aslam H. A., Ramashri T. and Ahsan M.I.A., A New Approach to Image Segmentation for Brain Tumor detection using Pillar K-means Algorithm, International Journal of Advanced Research in Computer and Communication Engineering, 2(3) (2013) 1429-1436.

15. Cebeci Z., Yildiz F., Comparison of K-Means and Fuzzy C-Means Algorithms on Different Cluster Structures, Journal of Agricultural Informatics, 6(3) (2015) 13-23.

16. El-Khamy E. S.,. Sadek A. R. A, El-Khoreby A. M., An Efficient Brain Mass Detection with Adaptive Clustered based Fuzzy C-Mean and Thresholding, IEEE International Conference on Signal and Image Processing Applications, 2015, pp. 429-433.

17. Poleti E., Veronese E., Calabrese M., Bertoldo A., Grisan E., Supervised Classification of Brain Tissues through Local Multi-scale Texture Analysis by Coupling DIR and FLAIR MR Sequences, Proceedings SPIE Medical Imaging, San Diego, California, United States, 8314 (2012) 83142T-83142T-7.

18. Pană L., Moldovanu S., Moraru L., "Differentiation of brain metastases in MRI image using the first- and second-order statistical features", Scientific Conference organized by the Doctoral Schools of "Dunărea de Jos" University of Galati (SCDS-UDJG), Galati, Romania, 2020.

19. Giavarina D., Understanding Bland Altman analysis, Biochem Medical, Zagreb, 25(2) (2015) 141-151.

20. Pană L., Moldovanu S., Moraru L.,Using grey-scale hit-or-miss transform for detection of isolated foreground pixels in cerebral RMN datasets, 19th International Multidisciplinary Scientific GeoConference SGEM, Section Advances in Biotechnology, Vienna, Austria, 2019.

21. Wan X., Wang W., Liu T. J. and Tong, Estimating the sample mean and standard deviation from the sample size, median, range and/or interquartile range, Journal BMC Medical Research Methodology, 14(Article number: 135) (2014).

22. Kalra A., Decoding the Bland-Altman plot: Basic review, Journal of the Practice of Cardiovascular Sciences, 3(1) (2017), 36-38. 